\title{
Evaluation of Drought Tolerance Indices and Nitrogen Fertilization for Some Groundnut (Arachis Hypogaea L.) Genotypes
}

\author{
Iancu Paula ${ }^{1}$, Păniță Ovidiu ${ }^{1}$ \& Soare Marin ${ }^{1}$ \\ ${ }^{1}$ Faculty of Agronomy, University of Craiova, Romania \\ Correspondence: Ovidiu Păniță, Faculty of Agronomy, University of Craiova, Libertății Street, No. 19, Romania. \\ E-mail: ovixfp@yahoo.com
}

Received: November 7, 2019 Accepted: December 2, 2019 Online Published: December 23, 2019

\begin{abstract}
Water is essential to maximize crop yield and quality. This natural resource has assumed huge importance, especially in the warmest areas, where drought and environmental degradation has affected agricultural production. In order to identify drought tolerance of some groundnut genotypes and to investigate the relationships between seed yield, quality and drought tolerance indices a study was made using 10 promising genotypes. The experiment was carried out during 2014-2017 and sowed under randomized block design with four replicates. It included three factors: two levels of irrigation ( $a_{1}$ - non - irrigated and $a_{2}$ - irrigated), two levels of fertilization $\left(b_{1}-\right.$ non-fertilized and $b_{2}-100$ active Nitrogen/ha) and genotype $\left(\mathrm{C}_{1}-\mathrm{C}_{10}\right)$. Seed yield depending on the influence of the factor, varied from $535.95 \mathrm{Kg} / \mathrm{ha}$ (non-irrigated) to $2020.95 \mathrm{Kg} / \mathrm{ha}$ (irrigated); from $1055.30 \mathrm{Kg} / \mathrm{ha}$ (non-fertilized) to 1501 $\mathrm{Kg} / \mathrm{ha}$ (fertilized) and from $1111.30 \mathrm{Kg} / \mathrm{ha}$ to $1388 \mathrm{Kg} / \mathrm{ha}$ depending on genotype. Same influence factors for protein content varied from $25.65 \%$ (irrigated) to $28.61 \%$ (non-irrigated); from $26.33 \%$ (non-fertilized) to $27.93 \%$ (fertilized) and from $25.59 \%$ to $28.52 \%$ depending on genotype. Stress susceptibility index (SSI) varied from 0.964 to 1.040; Stress Tolerance Index (STI) from 0.138 to 0.435 ; Mean Productivity (MP) from 883.5 to 1616.0; Geometric Mean Productivity (GMP) from 750.3 to 1332.7; Tolerance index (TOL) from 933.0 to 1844.0; Harmonic Mean (HM) from 637.2 to 1099.0; Yield Index (YI) 0.777 to 1.308 and Yield Stability Index (YSI) from 0.236 to 0.309 . High values of SSI, STI, YI, DI, RDI and SSPI indicate drought tolerance and those variants present high stability.
\end{abstract}

Keywords: fertilization, irrigation, PCA analysis, sandy soils

\section{Introduction}

Groundnut is a self-pollinating species and an annual herbaceous legume crop (Adinya et al., 2010). They have the potential to fix atmospheric nitrogen at the rate of 21 to $206 \mathrm{~kg} / \mathrm{ha}$ annually in soils through root nodule bacterium. They belongs to the genus Rhizobium, thus improves soil fertility (Yakubu et al., 2010). The amount of N-fixed in nodulated legumes is highly variable due to environmental conditions such as: solar irradiance (Izaguirre-Mayoral \& Sinclair, 2009, pp. 807-818), drought (Sinclair \& Vadez, 2012, pp. 501-512), soil temperature Divito \& Sadras, 2014, pp. 161-171) or other deficiencies.

Nitrogen is an important element for efficient production of groundnut. Adequate supply of nitrogen fertilizer is essential for obtaining better plants growth and yield. An application of $10 \mathrm{kgN} / \mathrm{ha}$ at the time of sowing is recommended for soils with moderate to low nitrogen (Fagbemigun \& Oguntola, 2019, pp. 86-94). Lack of nitrogen is observed when leaves turn into yellow and eventually, the plant's growth stops. In other cases, too much nitrogen is provided for the plant, it normally leads to watering of protoplasm and brittleness of the plant itself which would result in becoming vulnerable to diseases and pests (Awadalla \& Abbas, 2017, pp. 40-46).

Fertilizers and variety represents adequate agricultural practices to increase yield and quality to groundnut crop (Iancu Paula et al., 2014). The selection of high yielding genotypes is important for any crop productivity per unit area (Seadh, S.E. et al., 2017).

Although groundnuts are known to be more tolerant to drought stress than most other related plant species (Wan et al., 2014), drought seems to become one of the important abiotic stresses for crop productivity. High temperatures from the summer months can destroy many of the heat-sensitive species. Groundnuts are plants that grow on large areas in warm regions but may be affected by drought in certain phases of growth such as flowering and pods formation. 
Groundnut consumption is in continuous increase in Romania so adapting or creating new resistant/tolerance varieties is an important theme for researchers. Also, high yielding and of quality genotypes are preferred in the context of climate change. So, given the economic potential of groundnut, optimal fertilizer use, appropriate crop and soil fertility management practices could enhance productivity, food security and income of smallholder farmers from South region. Groundnut research experiences are set up in highly variable, low to medium rainfall and poor soil. Generally, the historical average annual rainfall is below $500 \mathrm{~mm}$ (occasionally $700 \mathrm{~mm}$ ). In agricultural policy terms, this region is considered to be less favored area (Soare et al., 2016).

The aim of this study is to investigate growth and yield response of groundnut genotypes as influenced by nitrogen fertilizer and irrigation.

\section{Method}

To evaluate yield and its quality, ten groundnut genotypes subjected to the influence of two irrigation levels and two different doses of Nitrogen, in a field experiment was conducted at Tamburesti Research Station, of University of Craiova, Romania, during 2014-2017, located at latitude of $44^{\circ} 1^{\prime} 40^{\prime \prime} \mathrm{N}$, longitude $23^{\circ} 56^{\prime} 9^{\prime \prime}$ E. The sandy soil contains $6-8 \%$ physical clay. The depth of the phreatic water is approximately at $7 \mathrm{~m}$ from the surface of dunes. The difference of the levels between dunes and interdunes varies between $2-\mathrm{m}$. The quantity of humus is low (0.3$0.5 \%$ at the sand of dune and 0.6-1.2 at the sand of interdune). The reaction of these sands varies from weak acid till neutral (D.H. $=6.3-7.1)$, while the total capacity of cation exchange is reduced $(3-5 \mathrm{~m}$ is at $100 / \mathrm{g}$ soil in the dune and $5-7 \mathrm{~m}$ is at $100 / \mathrm{g}$ soil at interdune). The sands are very poor in nitrogen and phosphor and mediocre supplied with potassium (0.03-0.05\% total nitrogen; $2-5 \mathrm{mg} / 100 \mathrm{~g}$ soil phosphor assimilable and approximately $12 \mathrm{mg} / 100 \mathrm{~g}$ soil assimilable potassium) (sand analysis made in Chemistry Laboratory of Faculty of Agronomy).

The experiment was carried out during 2014-2017 and sowed in randomized block design with three replicates. It included three factors: two levels of irrigation ( $a_{1}$ - non - irrigated and $a_{2}$ - irrigated), two levels of fertilization ( $b_{1}$ - non-fertilized and $\mathrm{b}_{2}-100$ active Nitrogen/ha) and genotype $\left(\mathrm{C}_{1}-\mathrm{C}_{10}\right)$. Data were statistically analyzed and means were compared by least significant differences (LSD), $\mathrm{P}=0.05$.

Drought tolerance indices were calculated using the formulas:

Stress susceptibility index $(\mathrm{SSI})=\left(1-\mathrm{Y}_{\mathrm{s}} / \mathrm{Y}_{\mathrm{p}}\right) /\left(1-\left(\overline{\mathrm{Y}}_{\mathrm{S}} \overline{\mathrm{Y}}_{\mathrm{p}}\right)\right.$

Tolerance $(\mathrm{TOL})=\mathrm{Y}_{\mathrm{p}}-\mathrm{Y}_{\mathrm{s}}$

Mean productivity $(\mathrm{MP})=\left(\mathrm{Y}_{\mathrm{p}}-\mathrm{Y}_{\mathrm{s}}\right) / 2$

Geometric mean productivity $(\mathrm{GMP})=\sqrt{\mathrm{Yp}-\mathrm{Ys}}$

Stress tolerance index $(\mathrm{STI})=\left(\mathrm{Y}_{\mathrm{s}} \times \mathrm{Y}_{\mathrm{p}}\right) / \overline{\mathrm{Y}}_{\mathrm{p}}^{2}$

Yield index $(\mathrm{YI})=\mathrm{Y}_{\mathrm{s}} / \overline{\mathrm{Y}}_{\mathrm{s}}$

Yield stability index $(\mathrm{YSI})=\mathrm{Y}_{\mathrm{s}} / \mathrm{Y}_{\mathrm{p}}$

Harmonic mean $(\mathrm{HM})=\left(2 \mathrm{xY}_{\mathrm{s}} \mathrm{x} \mathrm{Y}_{\mathrm{p}}\right) /\left(\mathrm{Y}_{\mathrm{p}}+\mathrm{Y}_{\mathrm{s}}\right)$

Sensitivity drought index $(\mathrm{SDI})=\left(\mathrm{Y}_{\mathrm{p}}-\mathrm{Y}_{\mathrm{s}}\right) / \mathrm{Y}_{\mathrm{p}}$

Drought resistance index (DI) $=\mathrm{Y}_{\mathrm{s}}{ }^{2} /\left(\bar{Y}_{\mathrm{s}} \mathrm{X} \mathrm{Y}_{\mathrm{p}}\right)$

Relative drought index $(\mathrm{RDI})=\left(\mathrm{Y}_{\mathrm{s}} / \mathrm{Y}_{\mathrm{p}}\right) /\left(\overline{\mathrm{Y}}_{\mathrm{s}} \overline{\mathrm{Y}}_{\mathrm{p}}\right)$

Stress susceptibility percentage index $(\mathrm{SSPI})=\left(\mathrm{Y}_{\mathrm{p}}-\mathrm{Y}_{\mathrm{s}}\right) \times 100 /\left(2 \times \overline{\mathrm{Y}}_{\mathrm{p}}\right)$

In order to analyze the yield potential under irrigation or drought conditions, the $Y_{p}$ values were considered the values of the $\mathrm{AxBxC}$ interaction from $\mathrm{A}_{2}$ level (irrigation), being of $\mathrm{a}_{2} \mathrm{~b}_{i} \mathrm{c}_{\mathrm{i}}$ type. The $\mathrm{Y}_{\mathrm{s}}$ values were considered the values of the $A x B x C$ interaction from $A_{1}$ level (drought), being of $a_{1} b_{i} c_{i}$ type. Correlation analysis yield, protein content and drought tolerance indices was performed to determine the best drought-tolerant genotypes and indices. Principal component analysis (PCA) was performed based on the analyzed indices. Both correlation and PCA were performed by IBM SPSS Version 2011 and MS Office Excel 2016.

\section{Results and Discussions}

Agriculture uses $11 \%$ of the world's land surface for crop production. It also uses $70 \%$ of all water withdrawn from aquifers, streams and lakes (FAO, 2011). For increasing agricultural yield it must use rationally doses of fertilizer as well as research for the possibility of water supplying.

Tamburesti Research Station is often subjected to unpredictable periods of water deficit and high temperatures in various stages of plants development. Mostly, the climate of the region is arid, characterized by cold winters and 
hot/dry summers. Three of the four experimentation years (2014-2017) were considered with drought, average temperatures being of $26^{\circ} \mathrm{C}$ and $400 \mathrm{~mm}$ rain water, with 4 degrees above, respective $300 \mathrm{~mm}$ under the average of the last 30 years.

Factor A: Analysis of yield under the influence of this factor, indicate that the mean of the irrigated variants recorded significant differences compared to the average of non-irrigated variants. In case of protein content, the average of variants in non-irrigated registered significant differences compared to the average of irrigation variants (table 1). It can see that drought can lead to a substantial decline in yield and this reduction depends on the genotype. Water stress causes a significant reduction in groundnut lines pod yield/ha and other characteristics (Nassar et al., 2018), so that water availability is a critical factor for a successful groundnut production.

Factor B: Analysis of yield from the average of the fertilized variants showed significant differences compared to the mean of non-fertilized variants. In the case of protein content, also the average of fertilized variants recorded significant differences compared to the mean of the non-fertilized variants.

Table 1. Factor A analysis influence on yield and protein content

\begin{tabular}{lcccc}
\hline & \multicolumn{2}{c}{$\mathrm{A}$} & \multicolumn{2}{c}{$\mathrm{B}$} \\
\cline { 2 - 5 } Factor Lever & Yield (kg/ha) & Protein (\%) & Yield (kg/ha) & Protein (\%) \\
\hline First level & $535.95 \pm 85.641^{\mathrm{b}}$ & $28.61 \pm 4.82^{\mathrm{a}}$ & $1055.30 \pm 599.83^{\mathrm{b}}$ & $26.33 \pm 2.05^{\mathrm{b}}$ \\
Second level & $2020.95 \pm 405.976^{\mathrm{a}}$ & $25.65 \pm 1.08^{\mathrm{b}}$ & $1501.60 \pm 912.34^{\mathrm{a}}$ & $27.93 \pm 1.70^{\mathrm{a}}$ \\
Mean & $1278.45 \pm 245.809$ & $27.13 \pm 2.95$ & $1278.45 \pm 756.09$ & $27.13 \pm 1.87$ \\
LSD 5\% & 35.70 & 2.61 & 76.440 & 0.673 \\
\hline
\end{tabular}

* Mean followed by similar letters in each column, are not significantly different at the $5 \%$ level of probability.

Applying nitrogen fertilizer dose of N90 twice, before sowing and in vegetation, leads to the obtaining of a very significant increase of production $1321 \mathrm{~kg} / \mathrm{ha}$ (Dima et al., 2013). Same authors reported a protein content ranging from $21.3 \%$ in the control variant unfertilized and $23.9 \%$ in the variant fertilized with N60P60K60 and nitrogen was given $1 / 3$ at sowing $+2 / 3$ in vegetation. The effects of $\mathrm{N}$ fertilization in oil seeds increase protein content but also have an adverse effect on oil content (Maheswari et al., 2017).

Romania is being placed in the north limit of cultivation areal of groundnut, where the interaction genotype $\mathrm{x}$ environment is very high, making difficult the identification of some cultivars easily adaptable to the sudden change of temperature which appears along vegetation period and to different levels of ensuring the heat factor from one year to another (Soare and Iancu, 2011).

As concern the method of irrigation, it seems that the application of micro-irrigation in groundnut has shown that the technique results in high water use efficiency, saves water, reduces fertilization requirement, provides better quality crop and higher pods yield (Jeetendra, K.S. et all., 2016).

Factor C: Analysis on yield of this factor leaded to a best result by genotype $\mathrm{C} 10$ which, along with the second genotype $\mathrm{C} 9$, recorded significant differences compared to the last 7 classified genotypes. In the analysis of factor $\mathrm{C}$ influence on protein content, the first classified genotype, $\mathrm{C} 1$, shows significant differences compared to all other analyzed genotypes (table 2). 
Table 2. Factor $\mathrm{C}$ analysis on yield and protein content

\begin{tabular}{|c|c|c|}
\hline $\mathrm{C}$ level $^{\text {Index }}$ & Yield (kg/ha) & Protein (\%) \\
\hline $\mathrm{C}_{1}$ & $1111.75 \pm 742.76^{\mathrm{f}}$ & $28.52 \pm 2.33^{\mathrm{a}}$ \\
\hline $\mathrm{C}_{2}$ & $1215.50 \pm 809.22^{\mathrm{e}}$ & $27.75 \pm 2.09^{b}$ \\
\hline $\mathrm{C}_{3}$ & $1232.25 \pm 813.72^{\mathrm{de}}$ & $27.50 \pm 2.18^{\mathrm{bc}}$ \\
\hline $\mathrm{C}_{4}$ & $1246.25 \pm 826.87^{\text {cde }}$ & $27.34 \pm 1.90^{\mathrm{bcd}}$ \\
\hline $\mathrm{C}_{5}$ & $1275.75 \pm 831.99^{\mathrm{cd}}$ & $27.24 \pm 1.88^{\mathrm{cd}}$ \\
\hline $\mathrm{C}_{6}$ & $1281.25 \pm 834.08^{\mathrm{cd}}$ & $27.05 \pm 2.04 \mathrm{de}$ \\
\hline $\mathrm{C}_{7}$ & $1304.25 \pm 844.81^{b c}$ & $26.92 \pm 1.97$ def \\
\hline $\mathrm{C}_{8}$ & $1351.25 \pm 857.26^{\mathrm{ab}}$ & $26.78+2.10^{\mathrm{e}}$ \\
\hline $\mathrm{C}_{9}$ & $1378.25 \pm 861.46^{\mathrm{a}}$ & $26.55 \pm 1.95^{\mathrm{f}}$ \\
\hline $\mathrm{C}_{10}$ & $1388.00 \pm 872.16^{\mathrm{a}}$ & $25.59 \pm 0.99^{\mathrm{f}}$ \\
\hline Mean & $1278.45 \pm 829.43$ & $27.12 \pm 1.94$ \\
\hline LSD $5 \%$ & 58.357 & 0.443 \\
\hline
\end{tabular}

* Mean followed by similar letters in each column, are not significantly different at the $5 \%$ level of probability

Referring to the analysis of the influence of factors $\mathrm{AxB}$ on yield, the differences between the variants are significant, the best result being obtained by $\mathrm{a} 2 \mathrm{~b} 2$ variant, fertilized and irrigated. The last classified variant is alb1, non-fertilized and non-irrigated variant. In the case of protein content, the differences between variants are significant, the best result being realized by a $1 \mathrm{~b} 2$ variant, non-irrigated and fertilized. The last classified variant is a2b1, non-fertilized and irrigated (table 3). Ghanbari et al., 2011, reported that yield, plant growth and nutrient uptake reduces under conditions of drought. Junjittakarn et al., 2013 sustain that water regimes can significantly affects nutrient uptakes of peanut, but selection of genotypes for high nutrient uptakes could be done under in any water regimes because the interactions between genotype and water regimes were not significant, showing the consistency of the nutrient uptakes across water regimes.

Table 3. Factors A and B interaction analysis influence on yield and protein content

\begin{tabular}{|c|c|c|c|c|}
\hline Factors level Indices & \multicolumn{2}{|c|}{ Yield (kg/ha) } & \multicolumn{2}{|c|}{ Protein $(\%)$} \\
\hline$x^{\text {A Iever }}$ & $\mathrm{a}_{1}$ & $a_{2}$ & $\mathrm{a}_{1}$ & $a_{2}$ \\
\hline $\mathrm{b}_{1}$ & $470.40 \pm 38.63^{\mathrm{d}}$ & $1640.20 \pm 151.27^{b}$ & $27.96 \pm 1.64^{\mathrm{b}}$ & $24.69 \pm 0.57^{\mathrm{d}}$ \\
\hline $\mathrm{b}_{2}$ & $601.50 \pm 74.49^{c}$ & $2401.70 \pm 141.62^{\mathrm{a}}$ & $29.25 \pm 1.45^{\mathrm{a}}$ & $26.61 \pm 0.39^{c}$ \\
\hline Mean & $1278.45 \pm 101.50$ & & $27.13 \pm 1.01$ & \\
\hline LSD $5 \%$ & 108.102 & & 0.952 & \\
\hline
\end{tabular}

* Mean followed by similar letters in each column, are not significantly different at the $5 \%$ level of probability

In the case of the analysis of the $\mathrm{AxC}$ interaction factor influence on the yield, between the first three variants $\left(\mathrm{a}_{2} \mathrm{C}_{10}, \mathrm{a}_{2} \mathrm{C}_{9}\right.$ and $\left.\mathrm{a}_{2} \mathrm{C}_{8}\right)$, genotypes with high potential under irrigation, there were not found significant differences, first two differentiating significant from the last 17 ranked variants. The last variant $\left(\mathrm{a}_{1} \mathrm{c}_{1}\right), \mathrm{C}_{10}$ genotype under irrigation, recorded significant negative differences compare with the first 12 ranked variants. In the case of protein content, the first ranked variant $\left(a_{1} c_{1}\right), C_{1}$ genotype under irrigation, recorded significant differences compare with all others variants. The last two ranked variants $\left(\mathrm{a}_{2} \mathrm{c}_{9}\right.$ and $\left.\mathrm{a}_{2} \mathrm{c}_{10}\right)$ recorded significant differences compare with the first 14 ranked variants (table 4). 
Table 4. Factors $\mathrm{A}$ and $\mathrm{C}$ interaction analysis influence on yield and protein content

\begin{tabular}{|c|c|c|c|c|}
\hline \multirow{2}{*}{ Factors lever } & \multicolumn{2}{|c|}{ Yield (kg) } & \multicolumn{2}{|c|}{ Protein $(\%)$} \\
\hline & $\mathrm{a}_{1}$ & $\mathrm{a}_{2}$ & $\mathrm{a}_{1}$ & $\mathrm{a}_{2}$ \\
\hline $\mathrm{c}_{1}$ & $466.00 \pm 91.09^{j}$ & $1757.50 \pm 452.39^{\mathrm{f}}$ & $30.61 \pm 0.97^{\mathrm{a}}$ & $26.45 \pm 0.84^{\text {fghi }}$ \\
\hline$c_{2}$ & $493.50 \pm 85.07^{\mathrm{ij}}$ & $1937.50 \pm 426.98^{\mathrm{e}}$ & $29.43 \pm 1.32^{b}$ & $26.09 \pm 1.11^{\text {fghi }}$ \\
\hline$c_{3}$ & $507.00 \pm 68.67^{\mathrm{hij}}$ & $1957.50 \pm 435.46^{\mathrm{de}}$ & $29.13 \pm 1.77^{\mathrm{bc}}$ & $25.89 \pm 1.02^{\mathrm{fghi}}$ \\
\hline $\mathrm{c}_{4}$ & $508.00 \pm 58.23^{\mathrm{hij}}$ & $1984.50 \pm 439.02^{\text {cde }}$ & $28.88 \pm 1.20^{\mathrm{cd}}$ & $25.82 \pm 0.97^{\text {ghij }}$ \\
\hline $\mathrm{c}_{5}$ & $527.00 \pm 59.20^{\text {ghij }}$ & $2024.50 \pm 416.96^{\text {cde }}$ & $28.75 \pm 1.21^{\mathrm{cd}}$ & $25.74 \pm 0.96^{\mathrm{hijk}}$ \\
\hline $\mathrm{c}_{6}$ & $530.00 \pm 60.91^{\text {ghij }}$ & $2032.50 \pm 415.08^{\mathrm{cd}}$ & $28.56 \pm 1.62^{\text {cde }}$ & $25.54 \pm 1.07^{\mathrm{ijk}}$ \\
\hline $\mathrm{c}_{7}$ & $552.00 \pm 80.28^{\mathrm{ghi}}$ & $2056.50 \pm 453.41^{\mathrm{bc}}$ & $28.38 \pm 1.50^{\mathrm{de}}$ & $25.47 \pm 1.13^{\mathrm{ijk}}$ \\
\hline $\mathrm{c}_{8}$ & $577.50 \pm 93.79^{\mathrm{gh}}$ & $2125.00 \pm 413.66^{\mathrm{ab}}$ & $28.31 \pm 1.59^{\mathrm{de}}$ & $25.26 \pm 1.27^{\mathrm{jk}}$ \\
\hline $\mathrm{c}_{9}$ & $600.00 \pm 105.64^{\mathrm{g}}$ & $2156.50 \pm 409.71^{\mathrm{a}}$ & $27.96 \pm 1.43^{\mathrm{e}}$ & $25.15 \pm 1.28^{\mathrm{k}}$ \\
\hline $\mathrm{c}_{10}$ & $598.50 \pm 114.14^{\mathrm{g}}$ & $2177.50 \pm 405.56^{\mathrm{a}}$ & $26.08 \pm 0.57^{\text {fghi }}$ & $25.12 \pm 1.14^{\mathrm{k}}$ \\
\hline Mean & $1278.450 \pm 254.26$ & & $27.13 \pm 1.20$ & \\
\hline LSD $5 \%$ & 82.529 & & 0.627 & \\
\hline
\end{tabular}

* Mean followed by similar letters in each column, are not significantly different at the $5 \%$ level of probability

In the case of the analysis of the $\mathrm{BxC}$ interaction factors influence on the yield, between the first four ranked variants $\left(b_{2} c_{10}, b_{2} c_{9}, b_{2} c_{8}\right.$ and $\left.b_{2} c_{7}\right)$ there were no statistical differences, the first two differentiating significant from the last 16 ranked variants. The last ranked variants $\left(b_{1} c_{1}\right)$ recorded significant negative differences compare with all others genotypes (table 5). Concerning the protein content, the first ranked variant $\left(b_{2} c_{1}\right)$ recorded significant differences compare with all others differences, except with the next two ranked variants $\left(b_{2} c_{3}\right.$ and $\left.b_{2} c_{3}\right)$. The last ranked variants $\left(b_{1} c_{10}\right)$ recorded significant negative differences compare with the first 18 ranked variants.

Table 5. Factors B and C interaction analysis influence on yield and protein content

\begin{tabular}{|c|c|c|c|c|}
\hline Indices & \multicolumn{2}{|c|}{ Yield (kg/ha) } & \multicolumn{2}{|c|}{ Protein $(\%)$} \\
\hline $\mathrm{C}_{\mathrm{C} \text { level }}$ B level & $\mathrm{b}_{1}$ & $\mathrm{~b}_{2}$ & $\mathrm{~b}_{1}$ & $\mathrm{~b}_{2}$ \\
\hline $\mathrm{c}_{1}$ & $883.50 \pm 513.685^{\mathrm{i}}$ & $1340.00 \pm 728.60^{\mathrm{d}}$ & $28.170 \pm 2.815^{\text {bcde }}$ & $28.880 \pm 1.951^{\mathrm{a}}$ \\
\hline $\mathrm{c}_{2}$ & $991.50 \pm 513.68^{\mathrm{h}}$ & $1439.50 \pm 908.20^{\mathrm{c}}$ & $26.750 \pm 1.919^{\text {gh }}$ & $28.765 \pm 1.875^{\mathrm{ab}}$ \\
\hline $\mathrm{c}_{3}$ & $1017.50 \pm 616.25^{\mathrm{h}}$ & $1447.00 \pm 969.77^{\mathrm{c}}$ & $26.520 \pm 1.769$ hi & $28.490 \pm 2.235^{\mathrm{abc}}$ \\
\hline $\mathrm{c}_{4}$ & $1028.50 \pm 618.72^{\mathrm{h}}$ & $1464.00 \pm 981.43^{\mathrm{bc}}$ & $26.475 \pm 1.800^{\mathrm{hi}}$ & $28.220 \pm 1.715^{\text {bcde }}$ \\
\hline $\mathrm{c}_{5}$ & $1061.50 \pm 635.69^{\mathrm{gh}}$ & $1490.00 \pm 993.11^{\mathrm{bc}}$ & $26.425 \pm 1.880^{\text {hijk }}$ & $28.060 \pm 1.632^{\text {cde }}$ \\
\hline $\mathrm{c}_{6}$ & $1068.50 \pm 640.01^{\text {fgh }}$ & $1494.00 \pm 1001.54^{\mathrm{bc}}$ & $26.200 \pm 2.237$ hijk & $27.900 \pm 1.577^{\text {cdef }}$ \\
\hline $\mathrm{c}_{7}$ & $1069.00 \pm 643.32^{\text {fgh }}$ & $1539.50 \pm 1004.00^{\mathrm{ab}}$ & $26.085 \pm 2.190^{\mathrm{ijk}}$ & $27.760 \pm 1.450^{\mathrm{def}}$ \\
\hline$c_{8}$ & $1125.50 \pm 643.81^{\text {efg }}$ & $1577.00 \pm 1011.35^{\mathrm{ab}}$ & $25.840 \pm 2.261^{\mathrm{jk}}$ & $27.725 \pm 1.584^{\mathrm{ef}}$ \\
\hline $\mathrm{c}_{9}$ & $1147.50 \pm 687.56^{\mathrm{ef}}$ & $1609.00 \pm 1010.80^{\mathrm{a}}$ & $25.695 \pm 2.224^{\mathrm{kl}}$ & $27.405 \pm 1.312^{\mathrm{fg}}$ \\
\hline $\mathrm{c}_{10}$ & $1160.00 \pm 708.47^{\mathrm{e}}$ & $1616.00 \pm 1001.45^{\mathrm{a}}$ & $25.115 \pm 1.129^{1}$ & $26.075 \pm 0.590^{\mathrm{ijk}}$ \\
\hline Mean & $1278.450+1008.88$ & & $27.128+1.81$ & \\
\hline LSD $5 \%$ & & & 0.627 & \\
\hline
\end{tabular}

*Mean followed by similar letters in each column, are not significantly different at the $5 \%$ level of probability 
Table 6 indicate the influence of the 3 factors on yield and the first ranking variants are the most productive genotypes, $\mathrm{C}_{7}, \mathrm{C}_{8}, \mathrm{C}_{9}$ and $\mathrm{C}_{10}$, under irrigation and with fertilization, those ones differentiating significantly from all other variants, except the next three ranked under them. The last ranking variants are all without fertilization and irrigation in order of their yield potential.

Table 6. Analysis of influence of the factors A, B and C interaction on yield and protein content

\begin{tabular}{|c|c|c|c|c|}
\hline \multirow{2}{*}{$\underbrace{\text { Factors level }}_{\text {BXC level }}$} & \multicolumn{2}{|c|}{ Yield (kg/ha) } & \multicolumn{2}{|c|}{ Protein $(\%)$} \\
\hline & $a_{1}$ & $\mathrm{a}_{2}$ & $\mathrm{a}_{1}$ & $\mathrm{a}_{2}$ \\
\hline $\mathrm{b}_{1} \mathrm{c}_{1}$ & $417.00+29.44^{18}$ & $1350.00 \pm 77.11^{12}$ & $30.64 \pm 1.20^{1}$ & $25.70 \pm 0.27^{15-16}$ \\
\hline $\mathrm{b}_{1} \mathrm{c}_{2}$ & $432.00 \pm 55.43^{18}$ & $1551.00 \pm 84.87^{11}$ & $28.40 \pm 1.00^{5-7}$ & $25.10 \pm 0.17^{16-17}$ \\
\hline$b_{1} c_{3}$ & $455.00 \pm 60.62^{17-18}$ & $1580.00 \pm 64.37^{10}$ & $28.05 \pm 0.83^{6-8}$ & $24.99 \pm 0.33^{16-18}$ \\
\hline $\mathrm{b}_{1} \mathrm{c}_{4}$ & $457.00 \pm 20.78^{17-18}$ & $1600.00 \pm 173.21^{10}$ & $28.00 \pm 1.06^{6-9}$ & $24.95 \pm 0.09^{16-18}$ \\
\hline $\mathrm{b}_{1} \mathrm{c}_{5}$ & $478.00 \pm 31.18^{16-18}$ & $1645.00 \pm 40.73^{9-10}$ & $27.95 \pm 1.32^{6-9}$ & $24.90 \pm 0.36^{16-18}$ \\
\hline $\mathrm{b}_{1} \mathrm{c}_{6}$ & $482.00 \pm 38.46^{16-18}$ & $1655.00 \pm 51.96^{9-10}$ & $27.82 \pm 2.15^{6-9}$ & $24.58 \pm 0.14^{17-19}$ \\
\hline $\mathrm{b}_{1} \mathrm{c}_{7}$ & $483.00 \pm 22.52^{16-18}$ & $1655.00 \pm 74.32^{9-10}$ & $27.68 \pm 2.04^{6-9}$ & $24.49 \pm 0.43^{17-19}$ \\
\hline $\mathrm{b}_{1} \mathrm{c}_{8}$ & $500.00 \pm 17.32^{16-18}$ & $1751.00 \pm 88.33^{8-9}$ & $27.57 \pm 1.94^{7-10}$ & $24.11 \pm 0.19^{18-19}$ \\
\hline $\mathrm{b}_{1} \mathrm{c}_{9}$ & $505.00 \pm 12.12^{16-18}$ & $1790.00+127.58^{8}$ & $27.39 \pm 1.91^{8-11}$ & $24.00 \pm 0.34^{19}$ \\
\hline $\mathrm{b}_{1} \mathrm{c}_{10}$ & $508.00 \pm 59.77^{16-18}$ & $1825.00 \pm 21.70^{7-8}$ & $27.35 \pm 0.19^{8-11}$ & $24.10 \pm 0.31^{18-19}$ \\
\hline $\mathrm{b}_{2} \mathrm{c}_{1}$ & $515.00 \pm 112.58^{16-18}$ & $2165.00 \pm 112.58^{6}$ & $30.57 \pm 0.95^{1}$ & $27.19 \pm 0.20^{8-12}$ \\
\hline $\mathrm{b}_{2} \mathrm{c}_{2}$ & $555.00 \pm 60.62^{15-17}$ & $2324.00 \pm 60.62^{5}$ & $30.45 \pm 0.43^{1}$ & $27.08 \pm 0.29^{9-13}$ \\
\hline $\mathrm{b}_{2} \mathrm{c}_{3}$ & $559.00 \pm 45.73^{15-17}$ & $2335.00 \pm 108.73^{5}$ & $30.20 \pm 1.91^{1-2}$ & $26.78 \pm 0.29^{10-14}$ \\
\hline $\mathrm{b}_{2} \mathrm{c}_{4}$ & $559.00 \pm 15.59^{15-17}$ & $2369.00 \pm 105.59^{4-5}$ & $29.76 \pm 0.40^{1-3}$ & $26.68 \pm 0.29^{10-14}$ \\
\hline $\mathrm{b}_{2} \mathrm{c}_{5}$ & $576.00 \pm 24.25^{15-16}$ & $2404.00 \pm 240.25^{3-5}$ & $29.54 \pm 0.07^{2-3}$ & $26.58 \pm 0.29^{11-15}$ \\
\hline $\mathrm{b}_{2} \mathrm{c}_{6}$ & $578.00 \pm 48.50^{15-16}$ & $2410.00 \pm 408.50^{2-5}$ & $29.30 \pm 0.52^{3-4}$ & $26.50 \pm 0.26^{11-15}$ \\
\hline $\mathrm{b}_{2} \mathrm{c}_{7}$ & $621.00 \pm 36.37^{14-15}$ & $2458.00 \pm 360.37^{1-4}$ & $29.07 \pm 0.02^{3-5}$ & $26.45 \pm 0.32^{12-15}$ \\
\hline $\mathrm{b}_{2} \mathrm{c}_{8}$ & $655.00 \pm 60.62^{14-15}$ & $2499.00 \pm 60.62^{1-3}$ & $29.05 \pm 0.97^{3-5}$ & $26.40 \pm 0.26^{12-15}$ \\
\hline $\mathrm{b}_{2} \mathrm{c}_{9}$ & $695.00 \pm 25.98^{14}$ & $2523.00 \pm 25.98^{1-2}$ & $28.52 \pm 0.71^{4-6}$ & $26.29 \pm 0.27^{13-15}$ \\
\hline $\mathrm{b}_{2} \mathrm{c}_{10}$ & $702.00 \pm 20.78^{13}$ & $2530.00 \pm 20.78^{1}$ & $26.02 \pm 0.90^{14-15}$ & $26.13 \pm 0.23^{14-15}$ \\
\hline Mean & \multicolumn{2}{|c|}{$1278.78 \pm 46.92$} & \multicolumn{2}{|c|}{$27.16 \pm 0.65$} \\
\hline $\operatorname{LSD} 5 \%$ & \multicolumn{2}{|c|}{116.714} & \multicolumn{2}{|c|}{0.900} \\
\hline
\end{tabular}

*Mean followed by similar numbers in each column, are not significantly different at the $5 \%$ level of probability

Regarding the influence of the three factors on the protein content, the first ranking variants are the ones of the $\mathrm{C}_{1}$ genotype with fertilization and no fertilization, respectively $\mathrm{C}_{2}, \mathrm{C}_{3}$ and $\mathrm{C}_{4}$ genotypes variants with fertilization. The last two variants are the ones of the $\mathrm{C}_{9}$ and $\mathrm{C}_{10}$ genotypes with no fertilization and under irrigation.

Drought indices are considered a measure to provide information about drought based on loss of yield in drought conditions in comparison to normal conditions and are used for screening drought tolerant genotypes. In order to analyze the yield potential both under irrigation or drought conditions, the $Y_{p}$ values were considered the values of the $A x B x C$ interaction from $A_{2}$ level (irrigation), being of $a_{2} b_{i} c_{i}$ type. The $Y_{s}$ values were considered the values of the $\mathrm{AxBxC}$ interaction from $\mathrm{A}_{1}$ level (drought), being of $\mathrm{a}_{1} \mathrm{~b}_{\mathrm{i}} \mathrm{c}_{\mathrm{i}}$ type. The values are shown in table 7.

Thus, the MP, GMP and HM show the yield potential under irrigation or drought conditions. In the comparative analysis, based on the absolute value of those indices it can be seen the variants with the highest yield potential both under irrigation and in drought conditions, high values emphasizing the variants with highest potential. TOL, 
YSI and SDI show the drought tolerance, in the comparative analysis based on the values of those indices, being able to identify the variants with the highest yield stability, lowest values indicated them. SSI, STI, YI, DI, RDI and SSPI show the drought tolerance of each variant reported on the average of the all variants, high values showing variants with high stability.

Table 7. Analysis of drought indexes values

\begin{tabular}{|c|c|c|c|c|c|c|c|c|c|c|c|c|c|c|c|c|}
\hline Index & $\underbrace{\frac{\partial}{0}}_{\lambda}$ & 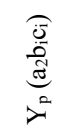 & $\underbrace{\frac{\hat{j}}{\tilde{J}}}_{0^{\infty}}$ & 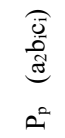 & $\overrightarrow{\widetilde{\Omega}}$ & $\stackrel{\ominus}{\ominus}$ & 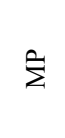 & $\sum_{j}^{e}$ & $\vec{\sim}$ & $\Xi$ & $\ddot{\sim}$ & 至 & $\vec{\omega}$ & $\overrightarrow{\bar{\theta}}$ & $\overrightarrow{2}$ & $\bar{a}$ \\
\hline $\mathrm{a}_{\mathrm{a}} \mathrm{b}_{1} \mathrm{c}_{1}$ & 417.0 & 1350.0 & 30.6 & 25.7 & 0.941 & 933.0 & 883.5 & 750.3 & 0.138 & 0.777 & 0.309 & 637.2 & 0.691 & 0.240 & 1.163 & 23.1 \\
\hline$a_{a} b_{1} c_{3}$ & 455.0 & 1580.0 & 28.1 & 25.0 & 0.969 & 1125.0 & 1017.5 & 847.9 & 0.176 & 0.848 & 0.288 & 706.5 & 0.712 & 0.244 & 1.085 & 27.8 \\
\hline $\mathrm{a}_{\mathrm{i}} \mathrm{b}_{1} \mathrm{c}_{4}$ & 457.0 & 1600.0 & 28.0 & 25.0 & 0.973 & 1143.0 & 1028.5 & 855.1 & 0.179 & 0.852 & 0.286 & 710.9 & 0.714 & 0.243 & 1.076 & 28.3 \\
\hline$a_{a} b_{1} c_{5}$ & 478.0 & 1645.0 & 28.0 & 24.9 & 0.966 & 1167.0 & 1061.5 & 886.7 & 0.193 & 0.891 & 0.291 & 740.8 & 0.709 & 0.259 & 1.094 & 28.9 \\
\hline$a_{a} b_{1} c_{8}$ & 500.0 & 1751.0 & 27.6 & 24.1 & 0.973 & 1251.0 & 1125.5 & 935.7 & 0.214 & 0.932 & 0.286 & 777.9 & 0.714 & 0.266 & 1.075 & 31.0 \\
\hline $\mathrm{a}_{\mathrm{a}} \mathrm{b}_{1} \mathrm{c}_{9}$ & 505.0 & 1790.0 & 27.4 & 24.0 & 0.977 & 1285.0 & 1147.5 & 950.8 & 0.221 & 0.941 & 0.282 & 787.8 & 0.718 & 0.266 & 1.063 & 31.8 \\
\hline$a_{b} b_{1} c_{10}$ & 508.0 & 1825.0 & 27.4 & 24.1 & 0.983 & 1317.0 & 1166.5 & 962.9 & 0.227 & 0.947 & 0.278 & 794.8 & 0.722 & 0.264 & 1.048 & 32.6 \\
\hline $\mathrm{a}_{\mathrm{a}} \mathrm{b}_{2} \mathrm{c}_{1}$ & 515.0 & 2165.0 & 30.6 & 27.2 & 1.038 & 1650.0 & 1340.0 & 1055.9 & 0.273 & 0.960 & 0.238 & 832.1 & 0.762 & 0.228 & 0.896 & 40.8 \\
\hline $\mathrm{a}_{\mathrm{a}} \mathrm{b}_{2} \mathrm{c}_{2}$ & 555.0 & 2324.0 & 30.5 & 27.1 & 1.036 & 1769.0 & 1439.5 & 1135.7 & 0.316 & 1.034 & 0.239 & 896.0 & 0.761 & 0.247 & 0.899 & 43.8 \\
\hline $\mathrm{a}_{3} \mathrm{~b}_{2} \mathrm{c}_{7}$ & 621.0 & 2458.0 & 29.1 & 26.5 & 1.018 & 1837.0 & 1539.5 & 1235.5 & 0.374 & 1.157 & 0.253 & 991.5 & 0.747 & 0.292 & 0.952 & 45.4 \\
\hline $\mathrm{a}_{1} \mathrm{~b}_{2} \mathrm{c}_{8}$ & 655.0 & 2499.0 & 29.1 & 26.4 & 1.005 & 1844.0 & 1577.0 & 1279.4 & 0.401 & 1.221 & 0.262 & 1037.9 & 0.738 & 0.320 & 0.987 & 45.6 \\
\hline $\mathrm{a}_{\mathrm{a}} \mathrm{b}_{2} \mathrm{c}_{9}$ & 695.0 & 2523.0 & 28.5 & 26.3 & 0.986 & 1828.0 & 1609.0 & 1324.2 & 0.429 & 1.295 & 0.275 & 1089.8 & 0.725 & 0.357 & 1.037 & 45.2 \\
\hline $\mathrm{a}_{\mathrm{i}} \mathrm{b}_{2} \mathrm{c}_{10}$ & 702.0 & 2530.0 & 26.0 & 26.1 & 0.984 & 1828.0 & 1616.0 & 1332.7 & 0.435 & 1.308 & 0.277 & 1099.0 & 0.723 & 0.363 & 1.045 & 45.2 \\
\hline Mean & 536.6 & 2021.0 & 28.7 & 25.7 & 0.995 & 1484.4 & 1278.8 & 1040.4 & 0.273 & 1.000 & 0.269 & 847.0 & 0.731 & 0.267 & 1.013 & 36.7 \\
\hline $\operatorname{Max}$ & 702.0 & 2530.0 & 30.6 & 27.2 & 1.040 & 1844.0 & 1616.0 & 1332.7 & 0.435 & 1.308 & 0.309 & 1099.0 & 0.764 & 0.363 & 1.163 & 45.6 \\
\hline Min & 417.0 & 1350.0 & 26.0 & 24.0 & 0.941 & 933.0 & 883.5 & 750.3 & 0.138 & 0.777 & 0.236 & 637.2 & 0.691 & 0.224 & 0.889 & 23.1 \\
\hline Std. dev. & 82.5 & 409.0 & 1.3 & 1.1 & 0.032 & 334.7 & 242.9 & 181.8 & 0.094 & 0.154 & 0.023 & 137.0 & 0.023 & 0.038 & 0.088 & 8.3 \\
\hline
\end{tabular}

Analyzing the correlation coefficients values for the drought tolerance indices, it can be seen that some of them show the same thing, because of the high correlation values between them. Those types of correlation with values almost equal with 1 or -1 were found between: YI and $\mathrm{Y}_{\mathrm{s}}$; SSI and YSI; SSI and SDI; SSI and RDI; TOL and SSPI; YSI and SDI; YSI and RDI and between SDI and RDI.

The yield recorded under irrigation conditions $\left(\mathrm{Y}_{\mathrm{p}}\right)$ is in very strong correlation with the next indices: Ys, SSI, TOL, MP, GMP, STI, Yi, HM, SDI and SSPI. The correlation between $Y_{p}$ and Ys suppose that the genotypes with 
high potential under irrigation conditions obtain the highest yield values on drought. $\mathrm{Y}_{\mathrm{s}}$ is in high strong correlation with: TOL, MP, GMP, STI, HM, DI and SSPI.

The indices that show high yield potential under irrigation conditions are in high positive correlation between them. There are strongly negative correlations between indices that show yield potential and indices that show yield stability on drought (table 8).

Table 8. Analysis of correlations between drought indices

\begin{tabular}{|c|c|c|c|c|c|c|c|c|c|c|c|c|c|c|c|}
\hline & $\begin{array}{c}\mathrm{Y}_{\mathrm{s}} \\
\left(\mathrm{a}_{1} \mathrm{~b}_{\mathrm{i}} \mathrm{c}_{\mathrm{i}}\right)\end{array}$ & $\begin{array}{c}\mathrm{Y}_{\mathrm{p}} \\
\left(\mathrm{a}_{2} \mathrm{~b}_{\mathrm{i}} \mathrm{c}_{\mathrm{i}}\right)\end{array}$ & $\begin{array}{c}P_{s} \\
\left(a_{1} b_{i} c_{i}\right)\end{array}$ & $\begin{array}{c}\mathrm{P}_{\mathrm{p}} \\
\left(\mathrm{a}_{2} \mathrm{~b}_{\mathrm{i}} \mathrm{c}_{\mathrm{i}}\right)\end{array}$ & SSI & TOL & MP & GMP & STI & YI & YSI & HM & SDI & DI & RDI \\
\hline$Y_{p}\left(a_{2}\right)$ & 0.919 & & & & & & & & & & & & & & \\
\hline$P_{s}\left(a_{1}\right)$ & -0.058 & 0.238 & & & & & & & & & & & & & \\
\hline $\mathrm{P}_{\mathrm{p}}\left(\mathrm{a}_{2}\right)$ & 0.551 & 0.759 & 0.715 & & & & & & & & & & & & \\
\hline SSI & 0.467 & 0.776 & 0.575 & 0.790 & & & & & & & & & & & \\
\hline TOL & 0.876 & 0.995 & 0.305 & 0.791 & 0.832 & & & & & & & & & & \\
\hline MP & 0.943 & 0.998 & 0.190 & 0.732 & 0.732 & 0.986 & & & & & & & & & \\
\hline GMP & 0.974 & 0.984 & 0.110 & 0.681 & 0.654 & 0.962 & 0.994 & & & & & & & & \\
\hline STI & 0.982 & 0.974 & 0.097 & 0.679 & 0.614 & 0.948 & 0.987 & 0.998 & & & & & & & \\
\hline Yi & 1.000 & 0.919 & -0.058 & 0.551 & 0.467 & 0.876 & 0.943 & 0.974 & 0.982 & & & & & & \\
\hline YSI & -0.467 & -0.776 & -0.575 & -0.790 & -1.000 & -0.832 & -0.732 & -0.654 & -0.614 & -0.467 & & & & & \\
\hline HM & 0.995 & 0.954 & 0.015 & 0.612 & 0.553 & 0.920 & 0.972 & 0.992 & 0.995 & 0.995 & -0.553 & & & & \\
\hline SDI & 0.467 & 0.776 & 0.575 & 0.790 & 1.000 & 0.832 & 0.732 & 0.654 & 0.614 & 0.467 & -1.000 & 0.553 & & & \\
\hline DI & 0.841 & 0.559 & -0.439 & 0.125 & -0.084 & 0.476 & 0.614 & 0.697 & 0.729 & 0.841 & 0.084 & 0.782 & -0.084 & & \\
\hline RDI & -0.467 & -0.776 & -0.575 & -0.790 & -1.000 & -0.832 & -0.732 & -0.654 & -0.614 & -0.467 & 1.000 & -0.553 & -1.000 & 0.084 & \\
\hline SSPI & 0.876 & 0.995 & 0.305 & 0.791 & 0.832 & 1.000 & 0.986 & 0.962 & 0.948 & 0.876 & -0.832 & 0.920 & 0.832 & 0.476 & -0.832 \\
\hline
\end{tabular}

$* \mathrm{P} 5 \%=0.44$

Significant positive correlations among YS and (YP, STI, YI, MP, YSI, GMP and HM), YP and (TOL, YR, STI, YI, MP, GMP and HM) reported others authors (Abejide et al., 2017). Same authors concluded that drought tolerance indices such as STI, MP, GMP, YI and HM which showed significantly high positive correlations with yield in both stressed and non-stressed conditions are good indicators which can be used for the screening and selection of drought tolerant genotypes also in Bambara groundnut. Also, Ilker et al., 2011 concluded that MP, GMP and STI values are convenient parameters to select high yielding wheat genotypes both in stress and nonstress conditions.

PCA analysis: Concerning the PCA analysis, the first component reaches over $74 \%$ from total variance, while the second component reaches over $21 \%$ from total variance. Together they have more than $95 \%$ from total variance.

The first component shows the yield potential both under irrigation and under drought conditions and low stability of the yield, the indices that quantify this potential being DI, YI, HM, GMP, STI, MP, SDI, SSI and SSPI. The second component shows the variants with high protein content both under irrigation or drought conditions, the indices that quantify this being YSI and RDI.

The correlation coefficients values of the two components and the analyzed indices are shown in table 9. All indices have at least 1 positive value for the correlation with the two components. In the case of the first component, 12 of the analyzed indices are in very high positive correlation, while only two indices, YSI and RDI, are in very high negative correlation with the second component. 
Table 9. Variation of correlation coefficients values between the two components and the analyzed indices

\begin{tabular}{|c|c|c|c|c|c|c|c|c|c|c|c|c|c|c|c|c|c|c|}
\hline & 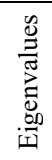 & $\begin{array}{l}\text { 岁 } \\
0^{\circ}\end{array}$ & 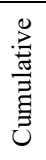 & $\underbrace{\frac{j}{0}}_{\lambda}$ & 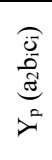 & $\underbrace{\frac{\partial j}{0}}_{e^{\infty}}$ & $\begin{array}{l}\text { ज्ञ } \\
\text { ज्ञ } \\
0^{2}\end{array}$ & $\overrightarrow{\tilde{n}}$ & $\hat{\Sigma}$ & $\sum_{0}^{B}$ & $\vec{E}$ & 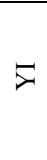 & $\bar{D}$ & $\sum$ & $\vec{\nabla}$ & $\overrightarrow{0}$ & $\overrightarrow{\widehat{\Omega}}$ & $\overrightarrow{\tilde{n}}$ \\
\hline $\mathrm{C}_{1}$ & $\stackrel{8}{=}$ & $\begin{array}{l}\infty \\
\stackrel{N}{+} \\
\stackrel{+}{+}\end{array}$ & $\begin{array}{l}\infty \\
\stackrel{\infty}{+} \\
\stackrel{N}{\sim}\end{array}$ & $\begin{array}{l}n \\
\infty \\
\infty \\
0\end{array}$ & $\begin{array}{l}\circ \\
\text { ลे }\end{array}$ & $\begin{array}{l}0 \\
\text { n! } \\
0\end{array}$ & $\begin{array}{l}n \\
\infty \\
\infty \\
0\end{array}$ & $\begin{array}{l}\stackrel{1}{~} \\
0 \\
0\end{array}$ & $\begin{array}{l}2 \\
\stackrel{\infty}{0} \\
0\end{array}$ & $\begin{array}{l}\hat{\circ} \\
\stackrel{0}{0}\end{array}$ & \begin{tabular}{l}
\multirow{2}{\alpha}{} \\
$\stackrel{0}{0}$
\end{tabular} & $\begin{array}{l}n \\
\infty \\
\infty \\
0\end{array}$ & $\begin{array}{l}\text { I } \\
\text { o. } \\
0 \\
0\end{array}$ & $\widehat{\widehat{\alpha}}$ & $\begin{array}{l}\stackrel{1}{0} \\
0 \\
0\end{array}$ & $\begin{array}{l}\stackrel{+}{+} \\
\dot{\sigma}\end{array}$ & $\begin{array}{l}\text { స్ } \\
\infty \\
0 \\
0\end{array}$ & $\stackrel{\alpha}{\circ}$ \\
\hline $\mathrm{C}_{2}$ & ले & $\frac{a}{\vec{\lambda}}$ & 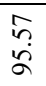 & $\begin{array}{l}+ \\
+ \\
+ \\
0\end{array}$ & $\begin{array}{l}\text { Õ } \\
\stackrel{0}{0} \\
0\end{array}$ & $\begin{array}{l}9 \\
0 \\
0 \\
0\end{array}$ & 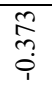 & 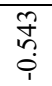 & $\stackrel{\infty}{\stackrel{\infty}{0}}$ & \begin{tabular}{l} 
ñ \\
\multirow{2}{0}{}
\end{tabular} & ते & $\begin{array}{l}\text { ț } \\
\stackrel{0}{0} \\
0\end{array}$ & 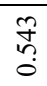 & 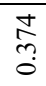 & $\begin{array}{l}\text { fo } \\
\stackrel{n}{?} \\
\stackrel{1}{1}\end{array}$ & $\begin{array}{l}8 \\
\dot{0} \\
\infty \\
0\end{array}$ & ?n & $\begin{array}{l}+ \\
0 \\
0 \\
0\end{array}$ \\
\hline
\end{tabular}

In the case of the second component, 9 of the analyzed indices have positive values, from which only DI is in strongly positive correlation with these one. Concerning the indices that are in negative correlation with the second component, only the correlation between one and Ps has a high negative value.

The variants ranking according to components score is shown in table 10 and figure 1 .

Table 10. The variants ranking according to components score based on PCA analysis

\begin{tabular}{|c|c|c|c|c|c|c|c|}
\hline \multicolumn{2}{|c|}{ The component sign } & \multirow{2}{*}{ Index } & \multicolumn{3}{|c|}{ Yield (kg/ha) } & \multicolumn{2}{|c|}{ Protein $(\%)$} \\
\hline $\mathrm{C}_{1}$ & $\mathrm{C}_{2}$ & & $\mathrm{a}_{1} \mathrm{~b}_{\mathrm{i}} \mathrm{c}_{\mathrm{i}}\left(\mathrm{Y}_{\mathrm{s}}\right)$ & $\mathrm{a}_{2} \mathrm{~b}_{\mathrm{i}} \mathrm{c}_{\mathrm{i}}\left(\mathrm{Y}_{\mathrm{p}}\right)$ & $\begin{array}{l}\mathrm{Y}_{\mathrm{p}}-\mathrm{Y}_{\mathrm{s}} \\
(\%)\end{array}$ & $\begin{array}{l}a_{1} b_{i} c_{i}\left(Y_{s}\right) \\
(\%)\end{array}$ & $\begin{array}{l}\mathrm{a}_{2} \mathrm{~b}_{\mathrm{i}} \mathrm{c}_{\mathrm{i}}\left(\mathrm{Y}_{\mathrm{p}}\right) \\
(\%)\end{array}$ \\
\hline \multirow{4}{*}{+} & \multirow{4}{*}{+} & $\mathrm{a}_{\mathrm{i}} \mathrm{b}_{2} \mathrm{C}_{7}$ & $621.00^{14-15}$ & $2458.00^{1-4}$ & 74.74 & $29.07^{3-5}$ & $26.45^{12-15}$ \\
\hline & & $\mathrm{a}_{\mathrm{i}} \mathrm{b}_{2} \mathrm{C}_{8}$ & $655.00^{14-15}$ & $2499.00^{1-3}$ & 73.79 & $29.05^{3-5}$ & $26.40^{12-15}$ \\
\hline & & $\mathrm{a}_{\mathrm{i}} \mathrm{b}_{2} \mathrm{C}_{9}$ & $695.00^{14}$ & $2523.00^{1-2}$ & 72.45 & $28.52^{4-6}$ & $26.29^{13-15}$ \\
\hline & & $\mathrm{a}_{\mathrm{i}} \mathrm{b}_{2} \mathrm{C}_{10}$ & $702.00^{13}$ & $2530.00^{1}$ & 72.25 & $26.02^{14-15}$ & $26.13^{14-15}$ \\
\hline Mean & & & 668.25 & 2502.5 & 73.31 & 28.17 & 26.32 \\
\hline \multirow{6}{*}{+} & \multirow{6}{*}{-} & $\mathrm{a}_{\mathrm{i}} \mathrm{b}_{2} \mathrm{C}_{1}$ & $515.00^{16-18}$ & $2165.00^{6}$ & 76.21 & $30.57^{1}$ & $27.19^{8-12}$ \\
\hline & & $\mathrm{a}_{\mathrm{i}} \mathrm{b}_{2} \mathrm{C}_{2}$ & $555.00^{15-17}$ & $2324.00^{5}$ & 76.12 & $30.45^{1}$ & $27.08^{9-13}$ \\
\hline & & $\mathrm{a}_{\mathrm{i}} \mathrm{b}_{2} \mathrm{C}_{3}$ & $559.00^{15-17}$ & $2335.00^{5}$ & 76.06 & $30.20^{1-2}$ & $26.78^{10-14}$ \\
\hline & & $\mathrm{a}_{\mathrm{i}} \mathrm{b}_{2} \mathrm{C}_{4}$ & $559.00^{15-17}$ & $2369.00^{4-5}$ & 76.40 & $29.76^{1-3}$ & $26.68^{10-14}$ \\
\hline & & $\mathrm{a}_{\mathrm{i}} \mathrm{b}_{2} \mathrm{C}_{5}$ & $576.00^{15-16}$ & $2404.00^{3-5}$ & 76.04 & $29.54^{2-3}$ & $26.58^{11-15}$ \\
\hline & & $\mathrm{a}_{\mathrm{i}} \mathrm{b}_{2} \mathrm{C}_{6}$ & $578.00^{15-16}$ & $2410.00^{2-5}$ & 76.02 & $29.30^{3-4}$ & $26.50^{11-15}$ \\
\hline Mean & & & 557 & 2334.5 & 76.14 & 29.97 & 26.80 \\
\hline \multirow{4}{*}{ - } & \multirow{4}{*}{-} & $\mathrm{a}_{\mathrm{i}} \mathrm{b}_{1} \mathrm{C}_{1}$ & $417.00^{18}$ & $1350.00^{12}$ & 69.11 & $30.64^{1}$ & $25.70^{15-16}$ \\
\hline & & $\mathrm{a}_{\mathrm{i}} \mathrm{b}_{1} \mathrm{C}_{2}$ & $432.00^{18}$ & $1551.00^{11}$ & 72.15 & $28.40^{-7}$ & $25.10^{16-17}$ \\
\hline & & $\mathrm{a}_{\mathrm{i}} \mathrm{b}_{1} \mathrm{C}_{3}$ & $455.00^{17-18}$ & $1580.00^{10}$ & 71.20 & $28.05^{6-8}$ & $24.99^{16-18}$ \\
\hline & & $\mathrm{b}_{1} \mathrm{C}_{4}$ & $457.00^{17-18}$ & $1600.00^{10}$ & 71.44 & $28.00^{6-9}$ & $24.95^{16-18}$ \\
\hline Mean & & & 440.25 & 1520.25 & 70.97 & 28.77 & 25.19 \\
\hline \multirow{6}{*}{ - } & \multirow{6}{*}{+} & $\mathrm{a}_{\mathrm{i}} \mathrm{b}_{1} \mathrm{C}_{5}$ & $478.00^{16-18}$ & $1645.00^{9-10}$ & 70.94 & $27.95^{6-9}$ & $24.90^{16-18}$ \\
\hline & & $\mathrm{a}_{\mathrm{i}} \mathrm{b}_{1} \mathrm{C}_{6}$ & $482.00^{16-18}$ & $1655.00^{9-10}$ & 70.88 & $27.82^{6-9}$ & $24.58^{17-19}$ \\
\hline & & $\mathrm{a}_{\mathrm{i}} \mathrm{b}_{1} \mathrm{C}_{7}$ & $483.00^{16-18}$ & $1655.00^{9-10}$ & 70.82 & $27.68^{6-9}$ & $24.49^{17-19}$ \\
\hline & & $\mathrm{a}_{\mathrm{i}} \mathrm{b}_{1} \mathrm{C}_{8}$ & $500.00^{16-18}$ & $1751.00^{8-9}$ & 71.44 & $27.57^{7-10}$ & $24.11^{18-19}$ \\
\hline & & $\mathrm{a}_{\mathrm{i}} \mathrm{b}_{1} \mathrm{C}_{9}$ & $505.00^{16-18}$ & $1790.00^{8}$ & 71.79 & $27.39^{8-11}$ & $24.00^{19}$ \\
\hline & & $\mathrm{a}_{\mathrm{i}} \mathrm{b}_{1} \mathrm{C}_{10}$ & $508.00^{16-18}$ & $1825.00^{7-8}$ & 72.16 & $27.35^{8-11}$ & $24.10^{18-19}$ \\
\hline Mean & & & 492.67 & 1720.17 & 71.34 & 27.63 & 24.36 \\
\hline
\end{tabular}

* Mean followed by similar numbers in each column, are not significantly different at the 5\% level of probability

Principal component analysis accounted $95.57 \%$ with the first PC of $74.38 \%$ whereas the second PC of $21.19 \%$. Regarding the variants ranking, the variants that have both components with high values are the variants where there were cropped $\mathrm{C}_{7}$ to $\mathrm{C}_{10}$ genotypes and there were applied the fertilization, those ones proving under irrigation and drought conditions the highest yield potential and the lowest drought tolerance and high protein content on drought and the lowest protein content under irrigation. 
The variants with first component with high value and second component with low value are the variants where there were cropped $C_{1}$ to $C_{6}$ genotypes and there were applied the fertilization, those ones proving under irrigation conditions high yield potential and on drought conditions low yield potential, low yield drought tolerance and the highest protein content on drought and low protein content under irrigation.

The variants that have both components with low values are the variants where there were cropped $\mathrm{C}_{1}$ to $\mathrm{C}_{4}$ genotypes and there were not applied fertilization, those ones proving under irrigation and drought conditions the lowest yield potential and the highest drought tolerance and the highest protein content. The variants with first component with low value and second component with high value are the variants where there were cropped $\mathrm{C}_{5}$ to $\mathrm{C}_{10}$ genotypes and there were not applied fertilization, proving under drought conditions the low yield potential and high yield potential under irrigation and medium protein content both under irrigation and drought conditions and also good yield drought tolerance.

In another experiment with winter wheat, principal component analysis was found of $96.063 \%$ from the variance which indicated limited variation in the response to reduced $\mathrm{N}$ fertilization and vice versa (Iancu Paula et al. 2019). Others authors also observed that the rank of genotypes for some studied traits under water stress changes from that under well watering conditions (Nassar et al. 2018).

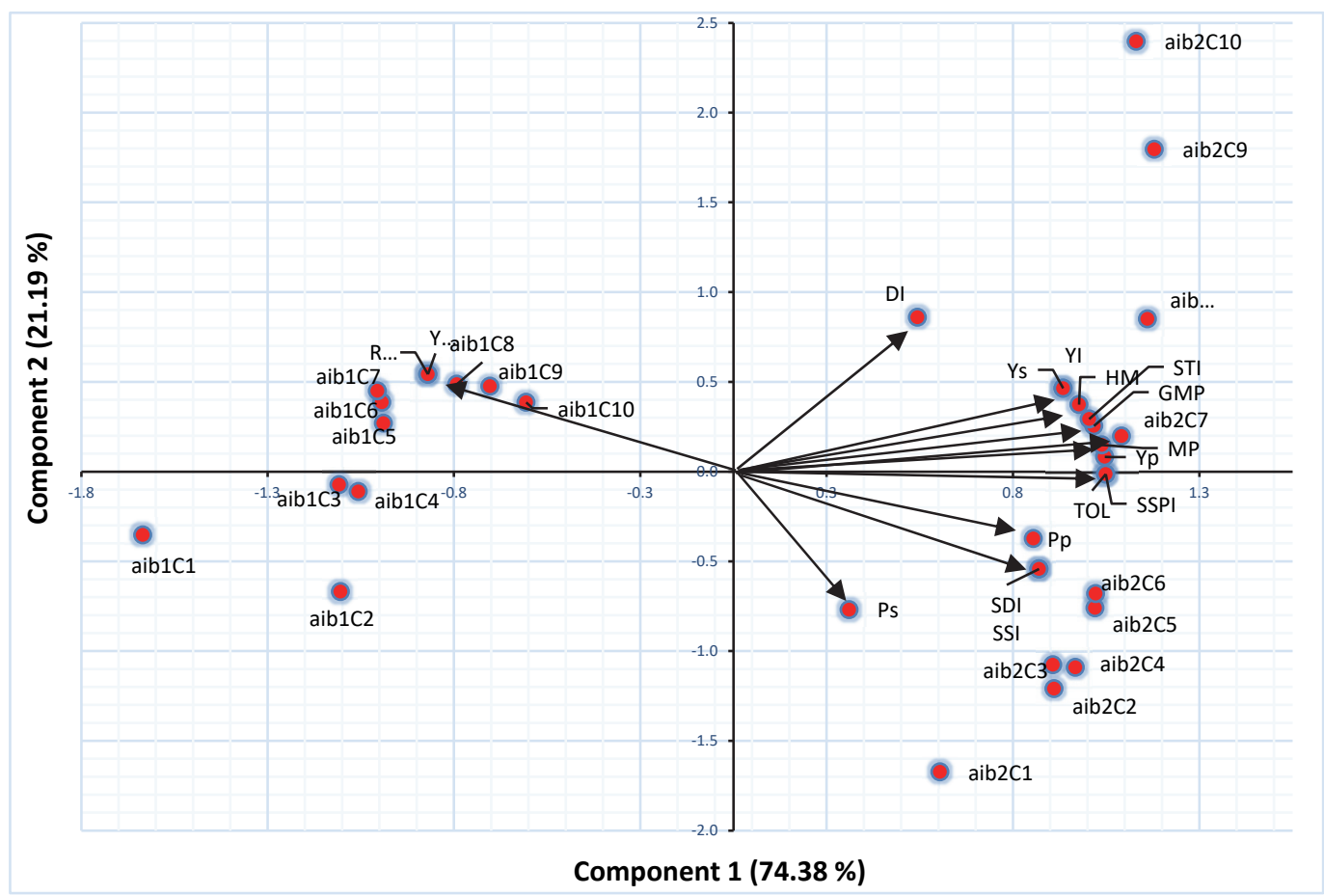

Figure 1. Biplot for drought or combine effect of all factors

\section{Conclusions}

For groundnut crops, water support is the most important factor, then fertilization and nitrogen factors. Some computed indices for the drought tolerance have very high values of the correlation coefficients, which proves that those ones are similar in determining the drought tolerance.

The yield under irrigation $\left(\mathrm{Y}_{\mathrm{p}}\right)$ is in positive correlation with the next indices: $\mathrm{Y}_{\mathrm{s}}$, SSI, TOL, MP, GMP, STI, Yi, $\mathrm{HM}$, SDI and SSPI.

The high correlation between Yp and Ys means that the genotypes with high yield potential under irrigation conditions have also high yield potential under drought conditions compare with the ones with low potential under irrigation.

The indices that indicate high yield potential under irrigation conditions are in high correlation between them.

On the PCA analysis, the first component shows the variants that have the highest yield potential both under irrigation or drought conditions and low tolerance under drought, those indices being: DI, YI, HM, GMP, STI, MP, 
SDI, SSI and SSPI. The second component shows the variants with high protein content both under irrigation or drought conditions, the indices that quantify this being YSI and RDI.

\section{References}

Abejide, D. R., Falusi, A. O., Adebola, M. O., Gana, A. S., Muhammad, L. M., \& Gado, A. A. (2017). Evaluation of drought tolerance indices in some nigerian bambara groundnut (Vigna subterranea L. VERDC.) landraces. IJABR, 8(2), 142-148.

Adinya, I. B., Enun, E. E., \& Ijoma, J. U. (2010). Exploring profitability potentials in groundnut production through agroforestry practices: a case study in Nigeria. Journal of Animal and Plant Sciences, 20(2), 123 - 131.

Awadalla, A. O., \& Abbas, M. T. (2017). Peanut (Arachis hypogaea L.). Yield and its components as affected by $\mathrm{N}$-fertilization and diazotroph inoculation in Toshka desert soil-South Valley-Egypt. Environ Risk Assess Remediat. 1(3), 40-46. https://doi.org/10.4066/2529-8046.100023

Dima, M., Croitoru, M., \& Drăghici, R. (2013). Research on application of NPK fertilizers in peanuts grown on sandy soils. Scientific Papers. Series A. Agronomy, LVI, 223-225.

Divito, G. A., \& Sadras, V. O. (2014). How do phosphorus, potassium and sulphur affect plant growth and biological nitrogen fixation in crop and pasture legumes? A meta-analysis. F. Crop Res., 156, 161171. https://doi.org/10.1016/j.fcr.2013.11.004

Fagbemigun, O. V., \& Oguntola, E. A. (2019). Effect of organomineral nitrogen starter fertilizer on the growth and yield of groundnut (Arachis hypogea L.). Advances in Plants \& Agriculture Research, 9(9), 86-94.

FAO. (2011). The state of the world's land and water resources for food and agriculture (SOLAW) - Managing systems at risk. Food and Agriculture Organization of the United Nations, Rome and Earthscan, London.

Ghanbari, A., Siahsar, B., Tovassoli, A., Esmaeilian, Y., \& Babaeian, M. (2011). Effect of uniconazole and cycocel on growth, yield and nutrient uptake of pearl millet under drought stress condition. Am-Euras J. Agric. Environ. Sci., 10, 857-862.

Iancu, P., Păniţă, O., Soare, M. (2019). Response of some new wheat genotypes to nitrogen fertilization and prospects of yield breeding based on yield elements. Romanian Agricultural Research, 36, 41-49.

Iancu, P., Soare, M., \& Matei, G. (2014). The influence of chemical fertilizers and soil upon the crop formation to groundnuts and the correlation with the phenomenon of withering. Analele Universității din Craiova, seria Agricultură - Montanologie - Cadastru (Annals of the University of Craiova - Agriculture, Montanology, Cadastre Serie), XLIV, 107-112.

Ilker, E., Tatar, O., Aykut, T. F., Tosun, M., \& Turk, J. (2011). Determination of tolerance level of some wheat genotypes to post anthesis drought. Turkish Journal of Field crops, 16(1), 59-63.

Izaguirre-Mayoral, M. L., \& Sinclair, T. R. (2009). Irradiance regulates genotype-specific responses of Rhizobium - nodulated soybean to increasing iron and two manganese concentrations in solution culture. J. Plant Physiol, 166(8), 807-818. https://doi.org/10.1016/j.jplph.2008.10.006

Jeetendra, K. S., Kumar, A., Kumar, V., Rani, S., \& Banerjee, A. (2016). Response of groundnut under micro irrigation - a review. Progressive Research - An International Journal, 11(Special-VII), 4455-4459.

Junjittakarn, J., Pimratch, S., Jogloy, S., Hotoon, W. N., Singkham, N., Vorasoot, N., Toomsan, B., Holbrook, C. C., \& Patanothai, A. (2013). Nutrient uptake of peanut genotypes under different water regimes. International Journal of Plant Production, 7(4), 677-692.

Maheswari, M., Murthy, A. N. G., \& Shanker, A. K. (2017). Nitrogen Nutrition in Crops and Its Importance in Crop Quality. Sources of Reactive Nitrogen, Environmental and Climate Effects, Management Options and Policies, 175-186. https://doi.org/10.1016/B978-0-12-811836-8.00012-4

Nassar, S. M. A., Al-Kady, A. M. A., \& El-Saka, Z. I. (2018). Effect of Drought Stress on Yield and Yield Components of 20 Peanut Genotypes Grown under Newly Reclaimed Soil. Egypt. J. Agro., 40(1), 45-58. https://doi.org/10.21608/agro.2018.2275.1088

Seadh, S. E., Abido, W. A. E., Abd, El-Al., A. N., \& Ibrahim, Z. A. A. (2017). Effect of NPK levels on productivity and quality of some groundnut genotypes under newly reclaimed sandy soils conditions. J. Plant Production, Mansoura Univ., 8(5), 605-609.

Sinclair, T. R., \& Vadez, V. (2012). The future of grain legumes in cropping systems. Crop Pasture Sci., 63, 501512. https://doi.org/10.1071/CP12128 
Soare, M., \& Iancu, P. (2011). Selection indices used to groundnut for choosing the initial material of breeding by irradiation. Analele Universitatii din Craiova, seria Agricultura - Montanologie - Cadastru, XLI(2), 231-236.

Soare, M., Iancu, P., \& Matei, G. (2016). Drought and its influence upon groundnut crop. SGEM $16^{\text {th }}$ International Multidisciplinary Scientific Geoconference. Advances in Biotechnology. Book 6(1), 517-524. https://doi.org/10.5593/sgem2016/b61/s25.068.

Wan, L., Wu, Y., Huang, J., Dai, X., Lei, Y., Yan, L., Jiang, H., Zhang, J., Varshney, R. K., \& Liao, B. (2014). Identification of ERF genes in peanuts and functional analysis of AhERF008 and AhERF019 in abiotic stress response. Funct. Integr. Genom., 14, 467-477. https://doi.org/10.1007/s10142-014-0381-4

Yakubu, H., Kwari, J. D., \& Tekwa, J. A. (2010). Nodulation and N2 - fixation by grain legumes as affected by Boron Fertilizers in Sudano - Sahelian Zone of North eastern Nigeria. American Eurasian Journal of Agriculture and Environmental Science, 8(5), 514-519.

\section{Copyrights}

Copyright for this article is retained by the author(s), with first publication rights granted to the journal.

This is an open-access article distributed under the terms and conditions of the Creative Commons Attribution license (http://creativecommons.org/licenses/by/4.0/). 